\title{
Bildungspolitik in den USA
}

\section{Eine historisch-institutionalistische Perspektive auf das Verhältnis von öffentlichen und privaten Bildungsinstitutionen ${ }^{1}$}

\begin{abstract}
Die im internationalen Vergleich überdurchschnittlich hohen Bildungsausgaben der USA sind vor allem auf die überdurchschnittlich bohen privaten Ausgaben im Bereich der Hochschulbildung zurückzuführen. Der vorliegende Aufsatz gibt eine Erklärung für diesen Befund, die aus drei Bausteinen besteht. Erstens hat der dezentrale Charakter des Bildungssystems und die damit einhergehende Wettbewerbsdynamik die Bildungsexpansion nicht gebremst, sondern befördert. Die frühe Demokratisierung und die relativ späte Zentralisierung der Verwaltungsbürokratie der USA erklären, wie es zu der Entstehung dieses dezentralen Systems gekommen ist. Der Wettbewerb im Bildungssystem hat im Primar- und Sekundarschulwesen zu einer Verdrängung der privaten durch öffentliche Schulen geführt, nicht jedoch im Hochschulsektor. Der zweite Baustein der Erklärung für die hohen Privatausgaben ist Regierungshandeln und -politik, die über Forschungs- und Studienförderung die Stellung der privaten Bildungsinstitutionen gefestigt haben. Hin₹u kommen Begünstigungen durch das Steuerrecht und eine ausgeprägte philanthropische Tradition. Das dritte Erkelärungselement ist das wohlfahrtsstaatliche Umfeld: Der liberale Arbeitsmarkt stellt sicher, dass sich Investitionen in die eigene Bildung und Ausbildung wegen der hohen Einkommensprämien lohnen. Der schwach ausgeprägte soziale Schutz macht diese Investitionen sogar erforderlich.
\end{abstract}

\section{Einleitung}

In der vergleichenden Wohlfahrtsstaaten-Forschung wurde dem US-amerikanischen Wohlfahrtsstaat traditionell ein ,residualer' bzw. ,liberaler' Charakter zugeschrieben (Titmuss 1958: 30-31; Esping-Andersen 1990) - eine Typologisierung, bei der der Vorwurf der Unterentwickeltheit mitklingt. In dieses Bild passt auch die Tatsache,

1 Die diesem Aufsatz zu Grunde liegende Forschungsarbeit wurde im Rahmen des von der DFG geförderten Projekts „Bildungsausgaben im internationalen Vergleich“ unter Leitung von Prof. Manfred G. Schmidt am Institut für Politische Wissenschaft der Universität Heidelberg sowie eines zweijährigen Studien- und Forschungsaufenthalts an der Kennedy School of Government der Harvard Universität durchgeführt. Zum Zweck des internationalen Vergleiches greift dieser Aufsatz auf die im Projekt aufgebaute Datenbasis zurück, deren Quellen vor allem öffentlich zugängliche Publikationen von einschlägigen internationalen Organisationen wie der OECD und der UNESCO sind. Trotz offensichtlicher Bemühungen der internationalen Organisationen zu Standardisierung ist die Datenqualität und Vergleichbarkeit, vor allem was frühe Datenpunkte angeht, leider nicht immer gegeben. Daher greift dieser Aufsatz auch auf historiographische Analysen und qualitative Methoden zurück. Ich danke Manfred G. Schmidt, Rita Nikolai, Frieder Wolf und Christine Trampusch für hilfreiche Anregungen und Kritik.

Konstanzer Online-Publikations-System (KOPS)

URL: http://nbn-resolving.de/urn:nbn:de:bsz:352-0-375028 
dass die öffentliche Sozialleistungsquote der USA mit 14,7\% des Bruttoinlandsproduktes (BIP) deutlich unter dem OECD-Durchschnitt von 20,8\% liegt (OECD 2006b). Neuere Forschungen zeigen allerdings, dass das in der klassischen vergleichenden Wohlfahrtsstaatenforschung vorherrschende Bild zwar nicht gänzlich unzutreffend ist, aber in einigen, möglicherweise entscheidenden Punkte revidiert bzw. ergänzt werden muss. Adema (2001) beispielsweise zeigt, dass die USA im internationalen Vergleich besser dastehen, wenn neben den reinen Sozialausgaben auch die Besteuerung von Sozialtransfers und sozialpolitisch motivierte Steuervergünstigungen berücksichtigt werden. Jacob Hacker (2002: 7) verweist außerdem auf die Bedeutung von privaten Sozialausgaben, die zum Beispiel in Form von betrieblichen Rentenoder Krankenversicherungsprogrammen vor allem von Arbeitgebern getragen werden. Für Hacker ist es somit weniger das Niveau der Gesamtsozialausgaben, das die USA von anderen entwickelten Wohlfahrtsstaaten unterscheidet, sondern deren Herkunft - nämlich weniger öffentliche, sondern stärker private Quellen.

Der ,residual erscheinende Charakter des US-amerikanischen Wohlfahrtsstaats wird auch in ein anderes Licht gerückt, wenn man ein Politikfeld in Augenschein nimmt, das in liberalen Wohlfahrtsstaaten - anders als häufig in Kontinentaleuropa zu einem umfassenden Bereich von Wohlfahrtspolitik gerechnet wird: die Bildungspolitik. Deutlicher noch als in den „klassischen“ Feldern der Politik der sozialen Sicherung fällt die herausgehobene Rolle privater Ausgaben bei der Finanzierung der Bildung auf: So lagen im Jahr 2003 die USA mit 5,4\% des BIP bei den öffentlichen Bildungsausgaben nur leicht über dem OECD-Durchschnitt (5,2\%). Die privaten Ausgaben waren mit 2,1 \% aber dreimal so hoch wie der Durchschnitt (0,7 \%, vgl. OECD 2006a: 205). Eine detaillierte Betrachtung nach Bildungssektoren zeigt, dass die hohen Privatausgaben vor allem auf den Hochschulbereich (tertiärer Bildungssektor) zurückzuführen sind (vgl. auch Busemeyer 2006): Im Primar-, Sekundar- und nicht-tertiären post-sekundären Bildungssektor ${ }^{2}$ liegen sowohl die öffentlichen als auch die privaten Bildungsausgaben der USA ungefähr im OECD-Durchschnitt. ${ }^{3}$ Im tertiären Bildungssektor liegen die öffentlichen Ausgaben $(1,2 \%$ des BIP) ebenfalls nur leicht über dem Durchschnitt $(1,1 \%)$, die privaten aber deutlich darüber $(2,9 \%$ bei einem Durchschnitt von $1,4 \%$, vgl. OECD 2006a: 206). Im Vergleich der relativen Position der USA bei den Sozialausgaben und den Bildungsausgaben fällt außerdem auf, dass die hohen privaten Sozialausgaben die unterdurchschnittlichen öffentlichen Ausgaben teilweise kompensieren können und die USA damit in eine Mittelfeldposition im Vergleich der Sozialausgaben anheben. Bei den Bildungsausgaben ist die relative Position der USA aber insgesamt nach oben verschoben: Die öffentlichen Ausgaben alleine begründen bereits eine solide Mittelfeldstellung der USA im Vergleich der OECD-Staaten, die hohen Privatausgaben befördern die Vereinigten Staaten aber an die Spitze der etablierten OECD-Mitglieder (vgl. auch Busemeyer 2006: 223).

2 Damit sind die Bereiche der Grundschulen (Primarsektor), weiterführenden Schulen (Sekundarbereich) und im Wesentlichen die berufliche Bildung (nicht-tertiärer, postsekundärer Sektor) gemeint.

3 Die öffentlichen Ausgaben für diesen Bildungssektor betragen 3,9\% des BIP (bei einem Durchschnitt von 3,6\%), die privaten $0,3 \%$ (genau auf OECD-Durchschnitt) - alle Daten aus OECD (2006a: 206). 
Das Bildungsausgabenmuster der USA weist damit im internationalen Vergleich zwei Auffälligkeiten auf, die erklärungsbedürftig sind: Zum einen zeigt sich, dass, gemessen an der Ausgabenintensität, der Bildung in den USA ein höherer Stellenwert zukommt als der Sozialpolitik (vgl. dazu auch Busemeyer 2006: 245). Zum anderen wird deutlich, dass die im internationalen Vergleich hohen Bildungsausgaben vor allem auf stark überdurchschnittliche private Ausgaben im Bereich der Hochschulbildung zurückzuführen sind.

Der Erklärung dieses Ausgabenmusters ist der größte Teil des vorliegenden Aufsatzes gewidmet. Hierbei ist zu bedenken, dass die privaten Bildungsausgaben aus verschiedenen Elementen zusammengesetzt sind, die sowohl das Angebot von Bildungsdienstleistungen durch Bildungsinstitutionen betreffen als auch die Nachfrage nach Bildung. Der internationale Vergleich zeigt, dass in den USA sowohl die Ausgaben der Hochschulinstitutionen (Angebotsseite) als auch die Zahlungsbereitschaft der Bildungskonsumenten (Nachfrageseite) hoch sind: Zum einen liegen, betrachtet man die jährlichen Ausgaben pro Student im Hochschulsektor, die USA mit 24.074 Dollar (PPP) an der Spitze, zusammen mit der Schweiz und mit weitem Abstand vor anderen europäischen Ländern (OECD 2006a: 186). Zum anderen sind die an USamerikanischen Hochschulen erhobenen Studiengebühren im internationalen Vergleich sehr hoch und können, je nach Institutionentyp, bis zu 40.000 Dollar pro Jahr betragen. Lediglich in Japan fallen höhere Studiengebühren an (Münch 2000: 90). Insofern stellt sich die Frage, warum und wie Hochschulinstitutionen in der Lage sind, die entsprechenden Ressourcen zu mobilisieren und warum Bildungsteilnehmer bereit sind, die hohen Studiengebühren zu zahlen, wenn sie im Rahmen von demokratischen Wahlprozessen langfristig die Verlagerung von gemeinschaftlich relevanten Aufgaben wie der Bildungspolitik in die öffentliche Verantwortung befördern könnten.

Im Folgenden sollen die zentralen Argumente des Aufsatzes kurz vorweggenommen und damit ein Ausblick auf die weitere Gliederung gegeben werden: Der erste Baustein zur Erklärung des US-amerikanischen Bildungsausgabenmusters ist der institutionelle Charakter des Bildungssystems, das sich durch seine ausgeprägte Dezentralität und eine damit verbundene hohe Wettbewerbsdynamik zwischen öffentlichen und privaten Bildungsinstitutionen, aber auch innerhalb des öffentlichen Sektors auszeichnet. Weil Bildungsdienstleistungen im Unterschied zu vielen sozialpolitischen Leistungen weniger auf umfassende Umverteilungs- oder Sozialversicherungssysteme angewiesen sind, hat der Wettbewerb um Schüler und Studenten im dezentralisierten Bildungssystem insgesamt die Bildungsexpansion beschleunigt. Ein umfassender Erklärungsversuch muss aber auch darlegen können, wie und warum es zu dem ausgeprägt dezentralen System gekommen ist und warum der Bildung vor der Sozialversicherungspolitik Vorrang eingeräumt wurde. Das relative Timing von makrosozialen Prozessen wie Demokratisierung und Zentralisierung der Verwaltungsbürokratie kann hier zur Erklärung beitragen (vgl. auch schon Heidenheimer 1981: 270).

Auffällig ist allerdings, dass die Bedeutung privater Bildungsinstitutionen im Hochschulsektor sehr viel stärker ausgeprägt ist als im Primar- und Sekundarschulwesen, obwohl beide Teilsysteme stark dezentralisiert waren und sind. Die Bildungsexpansion in der Primar- und Sekundarbildung ging einher mit einer Verdrängung von privaten durch öffentliche Institutionen. Dieser Verdrängungsprozess ist im Bereich der Hochschulbildung nicht so weit gegangen, so dass sich die Bedeutung 
privater Bildungsinstitutionen auf einem höheren Niveau konsolidiert hat. Regierungshandeln und -politik tritt daher als zweiter Erklärungsbaustein auf: Insbesondere wird argumentiert, dass in der kritischen Periode der ersten Nachkriegsjahrzehnte das gezielte Eingreifen der Bundesregierung über die Instrumente der Studien- und Forschungsförderung dazu beigetragen hat, dass private Hochschulinstitutionen ein weitaus größeres Gewicht behalten haben als Privatschulen im Primar- und Sekundarbildungssektor.

Der dritte Erklärungsbaustein ist das wohlfahrtsstaatliche Umfeld. Regierungspolitik wie Forschungszuwendungen an private Universitäten und die individuelle Förderung von Studierenden hatte nur deshalb langfristig Bestand, weil das wohlfahrtsstaatliche Umfeld der Bildungspolitik in den USA private Bildungsinvestitionen ermöglicht und notwendig gemacht hat. Dabei dürfen aber auch die sozialpolitischen Nebenwirkungen nicht vernachlässigt werden. Wenn in angelsächsischen, aber auch skandinavischen Wohlfahrtsstaaten Bildung eher als Teil eines umfassenden (aber nicht notwendigerweise universalen) wohlfahrtsstaatlichen Arrangements gesehen wird als in kontinentaleuropäischen Staaten (Allmendinger/Leibfried 2002: 288; Kaufmann 2001: 816), so stellt sich die Frage, inwiefern die Bildung auch sozialpolitische Funktionen wie den Ausgleich von sozialer Ungleichheit übernehmen kann.

\section{Wettbewerb der Bildungsinstitutionen im dezentralen Bildungssystem der USA}

Der dezentrale Charakter der politischen Institutionen, die hohe Vetospielerdichte sowie ein pluralistisches Interessenvermittlungssystem sind wohlbekannte Gründe für die verspätete Entwicklung und Expansion des US-amerikanischen Wohlfahrtsstaates (Obinger/Wagschal 2000; Obinger et al. 2005; Hacker 2002: 22). Für die Bildungspolitik muss der dezentrale und pluralistische Charakter der politischen Institutionen allerdings nicht unbedingt zur Expansionsbremse werden. Bei der Sozialpolitik ist zum Aufbau umfassender Sozialversicherungs- und Umverteilungssysteme ein gewisses $\mathrm{Ma}$ an Zentralisierung von administrativer und politischer Verantwortung sowie ein Mindestmaß an Solidarität zwangsläufig notwendig. Anders in der Bildungspolitik: Die Bildungsdienstleistung kann lokal erbracht werden und ist weniger abhängig von einem übergreifenden institutionellen Rahmen als die Sozialpolitik. Sicherlich werden in jüngerer Zeit Koordinierungs- und Steuerungsinstrumente auch und vor allem in den USA eingesetzt, um durch ,nationale Standards ${ }^{6}$ die Bildungsqualität zu steigern. ${ }^{4}$ Dies ändert aber nichts an der Tatsache, dass aufgrund der verminderten Koordinations- und Kooperationsnotwendigkeiten in einem dezentralisierten System eine Wettbewerbsdynamik zwischen Bildungsinstitutionen in Gang gesetzt werden kann, die insgesamt zu einer Expansion des Bildungssystems beiträgt. Dies betrifft prinzipiell öffentliche und private Bildungsinstitutionen, wobei die Unterscheidung, wie gezeigt werden wird, zu Anfang nicht besonders stark ausgeprägt war. Die expansive Wettbewerbsdynamik kann aber erklären, warum die anfängliche Schwerpunktsetzung auf Bildung so viele Ressourcen band, dass die sozialpolitische Expansion erst 
verspätet einsetzte, und die heute zu beobachtenden Ausgabenmuster von einer relativ höheren Bedeutung der Bildung zeugen. Ein Rückblick in die politische Geschichte der US-amerikanischen Bildungsfinanzierung soll diese Thesen unterstreichen.

\subsection{Frühe Demokratisierung, Religion und „Amerikanisierung“}

Als Ausgangspunkt und Voraussetzung zur Entstehung eines dezentralen Bildungssystems muss die frühe Demokratisierung der Vereinigten Staaten angesehen werden. Hierbei geht es nicht nur um die Tatsache, dass die USA auf nationaler Ebene bereits früh über demokratische Institutionen verfügten, sondern auch und vor allem darum, dass sich in den Siedlergemeinden auf lokaler Ebene fast zwangsläufig demokratische Entscheidungsverfahren aus praktischen Gründen etabliert hatten. Die gemeinschaftliche Erziehung der Kinder war von Anfang an eine wichtige Frage - auch, weil viele der frühen Siedlergruppen eine gemeinsame Religion oder Weltanschauung verband, die an die Kinder weitergegeben werden sollte.

Die frühe Demokratisierung hat auch dazu beigetragen, dass Bildung als Instrument des sozialen Aufstiegs von Anfang an im öffentlichen Diskurs den Charakter eines demokratischen Bürgerrechts hatte (Skocpol 1992: 23). Die Bedeutung der Bildung als demokratisches Bürgerrecht kommt auch in der einschlägigen Rechtsprechung der Gerichte zum Tragen: Im Jahre 1844 erkannte ein Gericht in Vermont zum Beispiel das Bürgerrecht auf den Besuch einer allgemeinen Schule an; 1926 verfügte ein Gericht in Washington, dass der College-Besuch zu einer „Notwendigkeit“ geworden sei, die jedem zustehe (Brubacher/Willis 1997: 258). Auch Horace Mann, der große Bildungsreformer des Common School Movement, war von der Existenz eines Bürgerrechtes auf Bildung überzeugt (Dichanz 1991: 28).

Preußen, das sich durch die frühe Einführung der allgemeinen Schulpflicht ebenfalls als bildungspolitischer Pionier etabliert hatte (Schneider 1982), wurde als Vorbild explizit abgelehnt, und zwar vor allem aufgrund seines undemokratischen Charakters (Jorgenson 1987: 10). Dieses Zitat aus Massachusetts von Mitte des 19. Jahrhunderts verdeutlicht dies zusätzlich:

A government system of education in Prussia is not inconsistent with the theory of Prussian society, for there all wisdom is supposed to be lodged in the government. But the thing is wholly inadmissible here [...] because, according to our theory, the people are supposed to be wiser than the government. [...] To entrust, then, the government with the power of determining the education which our children shall receive is entrusting our servant with the power to be our master (nach Nasaw 1979: 64).

Jenseits der Sphäre des öffentlichen Diskurses hat die frühe Demokratisierung letzten Endes dazu geführt, dass sich die Interessen der aufstrebenden Mittelschichten besser durchsetzen konnten. Die Privilegierung der Interessen der Mittelschichten war natürlich auch eine Folge der zunächst unvollständigen, ,defekten' Demokratisierung. Im Zuge des 19. Jahrhunderts und darüber hinaus verlief die Bildungsexpansion daher parallel zur fortschreitenden Demokratisierung. Konkrete Erscheinungsformen des Mittelschichten-Engagements waren Bildungsreformbewegungen wie das Common School Movement (Mitte des 19. Jahrhunderts), das die Universalisierung der Primarbil- 
dung zum Ziel hatte, sowie das Progressive Movement (Anfang des 20. Jahrhunderts), das eine umfassende Neuausrichtung und ,Modernisierung' der Bildung sowie eine Universalisierung der Sekundarbildung einforderte. Die Bewegungen waren dezentral, aber national in dem Sinne, dass es in vielen lokalen Gemeinden und Städten entsprechende Zusammenschlüsse von ,men and women of wealth, property, and social standing“ (Nasaw 1979: 30), wie Kirchenmänner, Kaufleute, Angestellte, Bankleute und Rechtsanwälte, gab, die sich den Zielen der Reform verschrieben und zumeist einen protestantischen Hintergrund hatten (Berube 1994: 1).

Die Mittelschichten waren an der Teilhabe der sich formierenden industriellen Ordnung interessiert. Durch Bildungsteilnahme vor allem im Sekundarschulbereich und im post-sekundären Sektor konnte eine effektive Abgrenzung zu den ,ungebildeten', einkommensschwachen Schichten erreicht werden sowie die Aneignung von Fertigkeiten, die im Zuge der Industrialisierung der entscheidende Hebel für sozialen Aufstieg sein konnten. Neben rein ökonomischen Interessen bestand aber auch ein genuines Interesse an der Verbesserung des Loses der Arbeiterkinder (Church 1976: 60). Die konservativ geprägten Reformer hatten dabei auch die Stärkung der sozialen Kontrolle im Blick, die sie durch den Wegfall der in prä-urbanen lokalen Gemeinden vorherrschenden sozialen Kontrollmechanismen als gefährdet betrachteten (Church 1976: 80; Kaestle 1983: 107; Nasaw 1979: 40). ${ }^{5}$ Dies war auch in Bezug auf die in dieser Zeitperiode zunehmenden Einwandererströme von Bedeutung. Die öffentlichen Schulen spielten bei der ,Sozialisierung' und ,Amerikanisierung ${ }^{6}$ der Immigranten eine wichtige Rolle, wobei die Einwanderergruppen je nach Herkunft und gefühltem Assimilierungsdruck' die Erziehung ihrer Kinder in eigens gegründeten Schulen vorzogen (Ralph/Rubinson 1980). Letzten Endes hatte die Bildungsreform auch eine religiöse Dimension: Zwar propagierte das Common School Movement offiziell die Trennung von Kirche und Staat, betrieb aber zur gleichen Zeit die Verankerung eines auf den allgemeinen Glaubensgrundsätzen des Protestantismus fußenden Curriculums (Jorgenson 1987: 20, 33), welches in zunehmenden Maße die Katholiken gegen das öffentliche Schulwesen in Stellung brachte. Nach dem Amerikanischen Bürgerkrieg dehnte sich die Bildungsreformbewegung mit großem missionarischen Eifer auf den Süden und Westen aus. Unter dem integrierenden Dach des „Evangelicism“ wurde „,a vast educational campaign to save the West from sin in general and Roman Catholicism in particular" (Cremin 1976: 48-49) gestartet.

\subsection{Schwache administrative Zentralisierung}

Neben der frühen Demokratisierung hat als zweiter Faktor auch das Fehlen einer umfassenden und durchstrukturierten Verwaltungsbürokratie auf der Ebene der

5 Hier zeigt sich eine interessante Parallele zu den unter Bismarck in Deutschland eingeleiteten Sozialpolitikmaßnahmen, bei denen die Kontrolle des Revolutionspotentials der Arbeiterschaft auch eine wichtige Motivation war (vgl. Schmidt 2005: 29). Den US-amerikanischen Reformern ging es jedoch durch die Schwerpunktsetzung auf die moralisch-soziale Erziehung der Arbeiterkinder auf um deren langfristige Emanzipation, während Bismarcks Sozialpolitik vor allem auch die Neutralisierung von Revolutionsgefahren zielte, ohne langfristig die gesellschaftliche Stellung der Nutznießer der Sozialpolitik zu verbessern. 
Bundesstaaten oder gar der Bundesregierung eine wichtige Rolle gespielt. Dadurch mussten viele Verwaltungs- und Dienstleistungen auf der lokalen Ebene erbracht werden, und es ist besonders bei der Bildungspolitik in der frühen Phase zu einer Vermischung zwischen der öffentlichen und der privaten Sphäre gekommen.

Bildungsinstitutionen - Schulen, aber auch Colleges - waren eng mit der sie tragenden lokalen und/oder weltanschaulichen Gemeinde verknüpft. Schon in der Kolonialzeit hatte das Schulgebäude (das ,little red school house“) nicht nur die Funktion einer Erziehungs- und Bildungsanstalt, sondern war auch ein sozialer Sammlungspunkt - auch, weil es oft das einzige öffentliche Gebäude in der Gemeinde war (Church 1976: 11; Kaestle 1983: 185). In den Frontierstaaten des Westens kam es ebenfalls zu einer faktischen, weil pragmatischen Verschmelzung von religiösen und allgemeinen Schulen (Jorgenson 1987: 53).

Auch die frühen Colleges des 19. Jahrhunderts waren teils öffentliche, teils private Institutionen. Primär dienten sie der Ausbildung von kirchlichem Nachwuchs, aber auch der Heranziehung gesellschaftlicher Führungskräfte (Jencks/Riesman 2002: 208; Lucas 1994: 105). Bei der Finanzierung der Colleges wurde zwar nicht der laufende Bildungsbetrieb subventioniert; Kapitalinvestitionen wie die Errichtung neuer Gebäude wurden aber häufig durch öffentliche Gelder unterstützt (Jencks/Riesman 2002: 257; Chambers 1962: 37). Dennoch mussten Studiengebühren entrichtet werden und die meisten Hochschulen waren offiziell mit einer religiösen Denomination affiliiert (Lucas 1994: 114).

Der religiöse Pluralismus, die offene Grenze nach Westen sowie die aufgrund des wenig ausgeprägten Bürokratismus relative Einfachheit der Gründung eines neuen Colleges führten zu einem regelrechten Bau-Boom. Bei College-Neugründungen standen zunächst religiöse Motive im Vordergrund, die zunehmend aber auch von ökonomischen und ethnischen Konfliktlinien ergänzt und überlagert wurden. Vor dem Amerikanischen Bürgerkrieg mussten allerdings ca. $80 \%$ der Neugründungen der vorangegangenen Jahrzehnte, die in die Hunderte gingen, wieder ihre Tore schließen (Pfnister 1962: 82).

Die fortschreitende Industrialisierung und deren Anforderungen an ausgebildete Arbeitskräfte führten um die Jahrhundertwende vom 19. ins 20. Jahrhundert eine neue Dimension des Wettbewerbs ein: Eine tiefgreifende Transformation der amerikanischen Hochschullandschaft läutete die Entstehung der „American University“ (Veysey 1965) ein, die in sehr viel stärkerem Maße forschungsorientiert war als die beschaulichen Land-Colleges der Vorkriegsperiode. Da die modernen Forschungsuniversitäten die Bedürfnisse der aufkeimenden Industrie sehr viel besser befriedigen konnten als die auf die Vermittlung klassizistischer Liberal Arts-Curricula ausgerichteten LandColleges, waren letztere einem harten Verdrängungswettbewerb ausgesetzt.

Die späte Bürokratisierung spielt auch beim Vormarsch der „American University“ eine Rolle. Weil die staatliche Bürokratie nicht im gleichen Maße durchgreifmächtig und administrativ durchstrukturiert war wie beispielsweise der Preußische Ordnungsstaat der damaligen Zeit, orientierten sich die aufkommenden Forschungsuniversitäten am einzig verbleibenden Rollenmodell einer modernen, nach Rationalitätskriterien ausgestalteten Verwaltungsorganisation: dem Wirtschaftsunternehmen (Lucas 1994: 188). Diese Entwicklung wurde befördert durch die großzügige Spendenbereitschaft der „Industriekapitäne“ wie Ezra Cornell, Cornelius Vanderbilt, Le- 
land Stanford oder Johns Hopkins, die dadurch nicht nur den jeweiligen Universitäten ihren Namen gaben, sondern durch die aktive Teilnahme in den universitären ,Aufsichtsräten“ („Board of Trustees“) den Transformationsprozess mitgestalten konnten (Brubacher/Willis 1997: 363; Veysey 1965: 350).

Wie fügen sich die einzelnen Puzzle-Teile zusammen und was tragen sie zur Erklärung der Fragestellung bei? Die frühe Demokratisierung trug dazu bei, dass der Bildungsexpansion Vorfahrt eingeräumt wurde, denn dies lag im Interesse der wichtigsten politischen Akteure, die auf Teilhabe in der sich formierenden ökonomischen Ordnung aus waren. Die schwache administrative Zentralisierung hat dazu beigetragen, dass Bildungsinstitutionen in einem engen Verhältnis zu der sie tragenden lokalen oder weltanschaulichen Gemeinde standen. Diese „local policy pre-emption“ (Leibfried et al. 2005: 323) hat eine weitergehende Zentralisierung stark gebremst. Dies hat aber die Bildungsexpansion nicht aufgehalten, sondern im Gegenteil noch gefördert. Die offene Grenze nach Westen und die pluralistische Gesellschaftsordnung der Vereinigten Staaten haben zu einem unkontrollierten Gründungsboom von Bildungsinstitutionen geführt.

Diese Wettbewerbslogik hat sich bis heute erhalten und ist ein wichtiger Bestandteil in der Erklärung der hohen Privatausgaben im Tertiärbildungssektor, und zwar vor allem auf der Angebotsseite. Erstens ist jede einmal gegründete Institution an ihrem Fortbestand interessiert. Die hohe Zahl von College-Schließungen vor dem Amerikanischen Bürgerkrieg, aber auch nach dem Zweiten Weltkrieg macht deutlich, dass der Wettbewerb die reale Option des Scheiterns beinhaltet. Dies treibt die Institutionen zu weiteren Anstrengungen an, und so ist es auch nicht verwunderlich, dass vor allem private Colleges und Universitäten schon früh versuchten, weitere Einnahmemöglichkeiten wie z.B. die Geldbeutel der Alumni oder den Verkauf von Dienstleistungen zu erschließen. Dies ist zweitens auch durch die Ausrichtung der amerikanischen Forschungsuniversitäten am Vorbild des Wirtschaftsunternehmens begünstigt worden. Dies erleichterte den privaten Bildungsinstitutionen auch den souveränen und wirtschaftlichen Umgang mit akquirierten Stiftungsgeldern („endowment" ${ }^{\circ}$. Drittens konnte der Wettbewerb und die damit einhergehende Autonomie der Bildungsinstitutionen auch als Chance genutzt werden, so dass sich das USamerikanische Hochschulsystem heute durch einen hohen Grad an Diversität auszeichnet und sich somit, alle erdenklichen Bildungsansprüche befriedigend, zu einem gewissen Teil seine Nachfrage selbst schaffen kann.

\subsection{Unterschiede zwischen Bildungssektoren}

Bislang hat die Argumentation nicht explizit zwischen öffentlichen und privaten Bildungsinstitutionen bzw. zwischen öffentlichen und privaten Ausgaben unterschieden. Der dezentrale Charakter des Bildungssystems betrifft beide Bereiche in gleicher Weise - allerdings mit durchaus unterschiedlichen Auswirkungen. Öffentliche Institutionen sind durch die Anbindung an die öffentlichen Haushalte prinzipiell ressourcenmächtiger als private Bildungseinrichtungen. Insofern wäre als ,natürliche' Entwicklung eine zunehmende Verdrängung von privaten Ausgaben und Institutionen durch öffentliche zu erwarten. Im Folgenden wird diese Entwicklungslogik ausführlicher dargestellt und gezeigt, dass entgegen den Erwartungen auffällige Unterschiede zwischen den Bildungssektoren zu beobachten sind. 
Die Bildungsexpansion in den einzelnen Bildungssektoren (Primar-, Sekundarund Hochschulbildung) durchläuft drei Entwicklungsphasen - natürlich zeitlich versetzt. Die erste Phase ist gekennzeichnet durch eine Vermischung der öffentlichen und privaten Verantwortung für die Bildung (die „little red school houses“ und die frühen „liberal arts colleges") und eine relativ beschränkte Bildungsexpansion.

In der zweiten Entwicklungsphase kommt es dann zu einer stärkeren quantitativen Expansion der Bildungsmöglichkeiten. Als politische Triebkraft hinter dieser Expansion treten die oben erwähnten nationalen, aber dezentralen Reformbewegungen auf - im Fall der Primarbildung das Common School Movement (Mitte des 19. Jahrhunderts) und im Fall der Sekundarbildung das Progressive Movement (Anfang des 20. Jahrhunderts). Diese Bewegungen werden vor allem von den Mittelschichten getragen, treten aber für eine gewisse Universalisierung der Bildungschancen ein. Eine Nebenfolge der einsetzenden Expansionsentwicklung ist die Anerkennung einer öffentlichen Verantwortung für Bildungspolitik und damit eine stärker werdende Trennung zwischen der öffentlichen und der privaten Sphäre.

Diese Trennung der beiden Verantwortlichkeiten ist wesentlich durch Gerichtsurteile vollzogen worden. Über lange Zeit hinweg wurden Schulen aus einer Vielzahl von öffentlichen (Zuschüsse der Bundesstaaten, Einnahmen aus Lotterien und Zöllen, etc.) und privaten (Schulgeld) Quellen finanziert. 1821 wurden dann die ersten privaten in rein öffentliche Schulen umgewandelt (Dresselhaus 1997: 199). Das Verfassungsgericht von Massachusetts prägte 1868 in einem Urteil die Definition einer öffentlichen Schule: Demnach ist eine öffentliche Schule 1. „supported by general taxation“, 2. „open to all free of expense“ und 3. „under the immediate control and superintendence of agents appointed by the voters of each town and city" (Jorgenson 1987: 7). Das dritte Element dieser Definition zeigt, wie wichtig das demokratische Element in der US-amerikanischen Bildungsgeschichte ist und warum die erwähnten sozialen Bewegungen über die dezentralisierten politisch-administrativen Institutionen des Bildungssystems einen solch starken Einfluss gewinnen konnten. Die Anbindung einer Bildungsinstitution an ein lokales, durch allgemeine Wahlen zu bestimmendes Gremium („school board“) wurde somit zum Unterscheidungskriterium zwischen öffentlichen und privaten Schulen.

Die Trennung zwischen öffentlichen und privaten Schulen wurde auch durch zunehmende Auseinandersetzungen zwischen der Minderheit der Katholiken und der protestantischen Mehrheit verschärft. Der Anteil der Katholiken an den Einwanderungswellen des 19. Jahrhunderts war höher als zuvor. Katholische Einwanderer protestierten gegen den in den öffentlichen Schulen dominierenden protestantischen Geist und gründeten eigene, private Schulen (Jorgenson 1987). Die protestantische Mehrheit war dementsprechend nicht bereit, die privaten Schulen durch öffentliche Gelder zu unterstützen.

Im Bereich der Hochschulbildung wurde die Separation der öffentlichen und privaten Sphäre durch die Dartmouth-Entscheidung (1819) des US-Verfassungsgerichts vollzogen. Ursprünglich ging es dabei um die Frage, ob die Legislative von New Hampshire das Recht habe, die Statuten des Dartmouth College zu verändern. Dies wurde vom Verfassungsgericht verneint, weil das College nicht als öffentliche Institution anzusehen sei und damit eindeutig den Status einer privaten Einrichtung erhielt. Das Verfassungsgericht erkannte nur dann Ausnahmen zu der Nicht-Ein- 
mischungsregel an, wenn eine akademische Einrichtung als öffentliche Körperschaft angelegt und mit öffentlichen Geldern finanziert sei (Lucas 1994: 116). Dieses Urteil führte somit eine strengere Teilung zwischen privaten und hoch-autonomen Hochschulen einerseits und öffentlichen, stärker an die politischen Institutionen angebundenen Einrichtungen andererseits ein.

Die Trennung zwischen öffentlicher und privater Sphäre läutete die dritte Entwicklungsphase ein: die zunehmende Verdrängung der privaten durch öffentliche Bildungseinrichtungen. Mit zunehmender Bildungsexpansion und zunehmender öffentlicher Verantwortung für die Bildungspolitik war dies quasi eine natürliche Folge. Die Finanzierung der öffentlichen Schulen wurde zunehmend über Grundsteuern (,property tax") geregelt, die von jedem Gemeindemitglied zu entrichten sind. Dadurch kamen vor allem die Mittelschichten in Bedrängnis: Sie waren zwar nicht wie die unteren Einkommensschichten gezwungen, ihre Kinder in öffentliche Armenschulen zu schicken (aus denen zum Beispiel in New York später das öffentliche Schulsystem hervorgegangen ist; Busemeyer 2006: 27). Sie konnten es sich aber im Unterschied zu den wohlhabenden Oberschichten nicht ohne Weiteres leisten, neben den zwangsläufig zu entrichtenden Grundsteuern auch teures Schulgeld zu bezahlen. Daher kam es in der zweiten Hälfte des 19. Jahrhunderts zu einer zunehmenden Verdrängung der privaten durch öffentliche Schulen (Kaestle 1983: 57).

Das Ergebnis dieses Verdrängungsprozesses ist, dass in der quantitativen Dimension private Schulen in der Primar- und Sekundarbildung in den USA heute kaum eine herausragende Stellung einnehmen. Weiter oben wurde schon auf die im internationalen Vergleich durchschnittliche Höhe der privaten Bildungsausgaben in den unteren Bildungssektoren hingewiesen. Auch der Anteil der Schüler in privaten Schulen kommt mit ca. $10 \%$ dem deutschen Wert sehr nahe (Münch 2000: 85). Ein Unterschied besteht allerdings im Grad der Abhängigkeit von staatlichen Stellen: USamerikanische Privatschulen werden weniger, quersubventioniert' ${ }^{\star}$ als europäische Privatschulen. Schüler an Privatschulen haben allerdings ein Anrecht auf bestimmte Bundesgelder zur Unterstützung des Transportes zur Schule, des Kaufs von Lehrbüchern oder bei der Verpflegung. Nichtsdestotrotz erfüllen Privatschulen im Primarund Sekundarbereich im Wesentlichen Nischenfunktionen: Zum einen handelt es sich hierbei um religiöse (vor allem katholische) Schulen und zum anderen um private Eliteschulen, die auf den Besuch von prestigeträchtigen Colleges vorbereiten (vgl. Coleman/Hoffer 1987: 30).

Auch im Hochschulbereich ist ein gewisser Verdrängungsprozess der privaten durch öffentliche Bildungsinstitutionen zu beobachten. Bereits oben wurde auf das ,College-Sterben“ im Verlauf des 19. Jahrhunderts verwiesen. Durch die Transformation der amerikanischen Hochschullandschaft um die Jahrhundertwende, die ja auch mit erheblichem Kapitaleinsatz von privaten Akteuren verbunden war, konnten private Institutionen wieder zeitweise einen Wettbewerbsvorteil erlangen. Im Zuge des 20. Jahrhunderts kam es dann zum Aufstieg der Community Colleges - tertiäre Bildungsinstitutionen, die vor allem lokale Bedürfnisse der weniger akademisch Begabten bedienten, dafür aber auch niedrige Studiengebühren verlangten (vgl. Bragg 2001). Besonders in der zweiten Hälfte des 20. Jahrhunderts engagierten sich zunehmend auch die Bundesstaaten in der Hochschulbildung und errichteten umfassende Hochschulsysteme, wobei das kalifornische System (der „California Master Plan“) 
durch die Breite seines Angebots vom Kindergarten über Zwei-Jahres-Colleges bis zur Elite-Uni in Berkeley als Vorbild diente (Nasaw 1979: 214). Öffentliche Hochschulinstitutionen waren und sind aufgrund ihres öffentlichen Charakters einem anderen Rechtfertigungsdruck ausgesetzt als private Institutionen und zeichnen sich daher durch insgesamt niedrigere Studiengebühren sowie einen offeneren Zugang aus (Fenton et al. 2001: 55). Prinzipiell waren private Hochschulen somit demselben Dilemma ausgesetzt wie private Schulen im Jahrhundert zuvor: Zur Rechtfertigung der höheren Studiengebühren mussten sie eine besonders hohe Qualität der Ausbildung bieten oder einem spezifischen weltanschaulichen Interesse genügen. Zunächst konnten private Institutionen einen Wettbewerbsvorteil aufrecht erhalten, indem sie im Unterschied zu öffentlichen Institutionen den Zugang selektiver gestalteten. So fiel der Anteil der an privaten Institutionen eingeschriebenen Studenten von ca. 50 \% (1919-1920) um jährlich einen Prozentpunkt auf 36 \% im Jahre 1964 (Jencks/Riesman 2002: 272). Die Bemühungen der privaten Hochschulinstitutionen konnten dennoch nicht verhindern, dass in der zweiten Hälfte des 20. Jahrhunderts vor allem private Institutionen ihre Tore schließen mussten und nicht öffentliche (Busemeyer 2006: 390).

Heute beträgt der Anteil der in privaten Bildungseinrichtungen im postsekundären Bereich Eingeschriebenen ca. 25 \% (NCES 2005, Tabelle 1), obwohl die privaten und öffentlichen Ausgaben in diesem Bildungssektor ungefähr gleich hoch sind (OECD 2006a: 206, siehe oben). Dabei ist natürlich zu bedenken, dass über Studiengebühren auch private Ausgaben an öffentliche Institutionen fließen, aber auch öffentliche Ausgaben über Forschungszuschüsse und Stipendienprogramme an private Institutionen. Dennoch liegen die Ausgaben pro Student an privaten Hochschulen gut $50 \%$ über den Ausgaben an öffentlichen Institutionen (NCES 2005, Tabelle 339; Busemeyer 2006: 188). Insofern stellt sich die Frage - und deren Beantwortung ist das Hauptanliegen dieses Aufsatzes -, warum private Hochschulinstitutionen in den USA eine solch herausgehobene Stellung haben. Deren Stellung ist auffällig im internationalen Vergleich (siehe Einleitung), aber auch im Vergleich der verschiedenen Bildungssektoren innerhalb des US-amerikanischen Bildungssystems. Die kurze Rekonstruktion der Geschichte der Bildungspolitik hat gezeigt, dass die ,natürliche' Entwicklungsdynamik der Bildungsexpansion in der Primar- und Sekundarschulbildung zu einer Verdrängung privater Institutionen geführt hat. Es konnte außerdem gezeigt werden, dass auch im Hochschulbereich Verdrängungstendenzen erkennbar waren. Die entscheidende Frage ist, warum diese Verdrängungstendenzen nicht so wie in der Primar- und Sekundarbildung zu einer Reduzierung der Bedeutung der privaten Institutionen auf Nischenfunktionen geführt haben, sondern im Ergebnis eine Konsolidierung des Systems auf einem wesentlich höheren Niveau im Hinblick auf die Bedeutung privater Hochschulinstitutionen zu beobachten ist. Im nächsten Abschnitt wird gezeigt, dass Regierungshandeln und -politik, vor allem der Bundesregierung, dabei eine wichtige Rolle gespielt haben.

\section{Die Rolle der Politik}

Wie bereits aus dem vorangegangen Abschnitt deutlich geworden sein dürfte, waren die ersten Jahrzehnte nach dem Zweiten Weltkrieg eine kritische Phase in der Ent- 
wicklung privater Bildungsinstitutionen, in der sich entschied, ob diese zu einem Nischendasein verdammt sein oder eine größere Rolle spielen würden. In dieser kritischen Phase der Pfadentscheidung spielten zwei Policy-Typen eine wichtige Rolle: Erstens sind dies Maßnahmen zur Unterstützung von Studenten und zweitens Maßnahmen zur Forschungsförderung. Beide Programme hatten nicht explizit die Stärkung privater Hochschulinstitutionen im Blick, sondern verfolgten hauptsächlich andere Ziele (Vermeidung von Arbeitslosigkeit einerseits und Stärkung der nationalen Forschungskapazitäten andererseits). Die Konsolidierung des privaten Hochschulsektors war aber ein durchaus nicht unerwünschter Nebeneffekt dieser Policies. Im Folgenden werden beide Policy-Ansätze und ihr Ursprung ausführlicher behandelt.

\subsection{Finanzielle Unterstützung für Studenten: die GI Bill}

Im Jahr 1943 wurde die GI Bill als Bundesgesetz verabschiedet. Im Rahmen der GI Bill wurden öffentliche Finanzmittel zur Unterstützung des Kaufs von Lehrbüchern, aber auch Zuschüsse zu Lebenshaltungskosten und Studiengebühren zur Verfügung gestellt. Dem Gesetzgeber ging es dabei aber nicht primär um die Herstellung von Chancengleichheit, sondern um die Vermeidung von drohender Arbeitslosigkeit, die nach der Rückkehr der Weltkriegs-Veteranen erwartet wurde ${ }^{6}$ (Ravitch 1983: 12 13; Nasaw 1979: 173, 175).

Im Hinblick auf unsere Forschungsfrage fallen zwei Eigenheiten des PolicyDesigns auf (zum Folgenden: Ravitch 1983): Erstens wurden die Finanzmittel nicht an Institutionen, sondern an Individuen gebunden, die dadurch in der Entscheidung darüber, welche Bildungsinstitution sie besuchen wollten, völlig frei blieben. Zweitens blieb auch die Autonomie der Hochschulen unangetastet, denn sie konnten selbst entscheiden, ob sie einen Kandidaten zulassen wollten oder nicht. Diese beiden Eigenheiten des Policy-Designs zeigen, dass eine Förderung der privaten Institution nicht primäres Ziel des Gesetzes war - aber eben auch nicht eine Förderung der Expansion der öffentlichen Institutionen, die zum Beispiel dadurch möglich gewesen wäre, dass man spezielle Studienplätze für Veteranen an öffentlichen Hochschulen eingerichtet hätte. Dass private Hochschulen faktisch von der GI Bill profitieren konnten, lag daran, dass sie sich durch selektiven Zugang und die konsequente Ausrichtung auf Forschung anstelle der reinen Lehre in den 1940er Jahren einen Wettbewerbsvorteil gegenüber öffentlichen Hochschulen erarbeitet hatten.

Dieser Vorteil wäre aller Wahrscheinlichkeit nach ohne die GI Bill und ähnlich gestrickte, sich anschließende Förderprogramme nur temporärer Natur gewesen und hätte langfristig zu einer Konsolidierung auf niedrigerem Niveau beigetragen. Die öffentliche Förderung, die Weltkriegsveteranen das Studieren an privaten Institutionen ermöglichte, hat somit vor allem zur quantitativen Expansion der privaten Bildungsalternativen beigetragen. Diese quantitative Wirkung der GI Bill ist beträchtlich: Insgesamt 2,2 Millionen Veteranen besuchten mit Förderung aus der GI Bill Institutionen der höheren Bildung; es wird geschätzt, dass von diesen ca. 20 bis $25 \%$ ohne das Gesetz kein College hätten besuchen können (Nasaw 1979: 180; Ravitch 1983: 14). Entgegen ursprünglicher Befürchtungen haben Veteranen es außerdem gut ver- 
standen, die ihnen gebotene Chance zu nutzen und sich durch überdurchschnittliche akademische Leistungen und eine hohe Arbeitsbereitschaft auszuzeichnen (Ravitch 1983: 14), so dass sie auf diese Weise zur Vergrößerung des Wettbewerbsvorteils der privaten Institutionen beitrugen.

Die Politik der Bundesregierung zur Unterstützung und Förderung von Studenten orientierte sich auch in den folgenden Jahren an den Grundprinzipien individuelle Förderung und autonome Hochschulwahl. In den 1960er und 1970er Jahren sind mit dem Higher Education Act und den Education Amendments weitere Stipendienund Kreditprogramme ins Leben gerufen worden, die sowohl Studenten an öffentlichen wie an privaten Institutionen zu Gute kommen. ${ }^{7}$ Der Taxpayer Relief Act von 1997 setzt leicht andere Schwerpunkte, weil er Bildungsinvestitionen über Steuererleichterungen fördert. Ein solches Instrument kommt vor allem oberen Einkommensschichten zu Gute (McKeown-Moak 2001: 12), aber es setzt dennoch weiterhin auf der individuellen Ebene an und fördert tendenziell sogar stärker den Besuch von privaten Institutionen, weil die Studiengebühren dort höher sind (und damit auch die Steuererleichterungen).

\subsection{Finanzielle Unterstützung von Hochschulen: Forschungsförderung}

Im angespannten Umfeld des Kalten Kriegs der 1950er Jahre unternahm die US-Bundesregierung erhebliche Anstrengungen zur Förderung von Forschungsaktivitäten sowohl in Unternehmen als auch in Universitäten. Weil die privaten Universitäten sich im Zuge der Transformation zur „American University“ bereits früh von der Lehre der Forschung zugewandt hatten, hatten sie auch hier einen Wettbewerbsvorteil, der ohne die zwischen öffentlichen und privaten Einrichtungen nicht diskriminierende Forschungsförderungspolitik der Bundesregierung allerdings wahrscheinlich ebenfalls temporär geblieben wäre. Jedenfalls hat auch hier die Politik der Bundesregierung öffentliche Institutionen nicht systematisch bevorzugt.

Einige Zahlen dienen der Verdeutlichung dieses Befundes: Die Bundesregierung war in der Forschungsförderung der entscheidende Akteur, auf den Ende der 1940er Jahre ca. $80 \%$ der nationalen Ausgaben für Forschung und Entwicklung zurückgingen (Lucas 1994: 232). In den ersten Nachkriegsjahrzehnten sind die öffentlichen Ausgaben für Forschungsförderung an Universitäten steil angestiegen von 6,2 Mio. (1938) auf 760 Mio. Dollar (1963) (Nasaw 1979: 187). Zwischen 1940 und 1964 stiegen die Bundesforschungsausgaben jährlich um $25 \%$ an (ebd.). Hinzu kamen Steuersubventionen für Forschungsförderung von Unternehmen an Universitäten, was auch deshalb bedeutend ist, weil fast alle Zuwendungen aus der Wirtschaft an private Colleges und Universitäten flossen (Nasaw 1979: 194).

Auch heute noch zeigen sich im Einnahmeprofil von forschungsorientierten öffentlichen und privaten Universitäten starke Ähnlichkeiten (vgl. zum Folgenden Bu-

7 Im Wesentlichen gibt es drei unterschiedliche Unterstützungsprogramme: die „Pell Grants“ sind nicht-rückzahlbare Zuschüsse oder Stipendien, während die „Perkins Loans“ über die individuellen Uni-Verwaltungen verteilt werden (aber dennoch größtenteils von der Bundesregierung finanziert sind). Hinzu kommen die „Stafford Loans“, bei denen die Bundesregierung eine Bürgschaft übernimmt (vgl. Peters 2004: 335; Choy 2004: 3-4). 
semeyer 2006: 192-195). Natürlich ist der Anteil von Zuschüssen aus den Haushalten der Bundesstaaten bei öffentlichen Institutionen höher. Hier schlägt die durch die Dartmouth-Entscheidung in Gang gesetzte Trennung zwischen öffentlichen und privaten Hochschulen durch, so dass private Institutionen die fehlenden Zuschüsse der Bundesstaaten durch höhere Einnahmen aus Studiengebühren, privaten Schenkungen und Einkünften aus Investitionen ausgleichen müssen. Was die Bedeutung der (öffentlichen) Forschungsförderung durch die Bundesregierung angeht, so ist das Profil von öffentlichen und privaten Institutionen allerdings relativ ähnlich: Der Anteil von Zuwendungen von der Bundesebene liegt bei beiden im Rahmen von 10 bis $15 \%$ der Gesamteinnahmen. Wenn man bedenkt, dass öffentliche Institutionen wegen des höheren Anteils an Studenten prinzipiell mehr Bundesmittel für individuelle Studienförderung bekommen, dann wird deutlich, dass die Forschungsförderung von privaten Universitäten durch die Bundesregierung beachtlich ist. Nach den Statistiken der National Science Foundation flossen 2004 insgesamt 11 Mrd. Dollar an Bundesmitteln zur Forschungsförderung an private Universitäten und Colleges und $17 \mathrm{Mrd}$. Dollar an öffentliche Einrichtungen (NSF 2004: Tabellen 8 und 11). Insofern hat auch die Bundesforschungsförderung durch ihren nicht-diskriminatorischen Ansatz dazu beigetragen, dass private Hochschulen ihre Wettbewerbsposition halten konnten.

\subsection{Steuerliche Begünstigungen und Philanthropie}

Keine Erläuterung der US-amerikanischen Bildungsausgaben kommt ohne einen Hinweis auf die Bedeutung der hohen Spendenbereitschaft und deren steuerlich günstige Behandlung aus. In der Tat sind private Zuwendungen an Hochschulen vollständig steuerabzugsfähig (Wright 2002) und das Stiftungs- und Erbschaftsrecht begünstigt die Vererbung an gemeinnützige Stiftungen (vgl. Beckert 2004). Hochschulen sind außerdem als Empfänger von Spenden äußerst beliebt: Hinter religiösen Gruppierungen und Kirchen (43,6 \% der Gesamtspendenmenge) sind sie die zweitwichtigste Empfängergruppe für Spenden (14,1\%) (Wright 2002). Nichtsdestotrotz spielen private Schenkungen und Zuschüsse als Einnahmequelle für öffentliche Institutionen (ca. $5 \%$ der Einnahmen) eine geringere Rolle als für private Institutionen (über $15 \%$ der Einnahmen) (Busemeyer 2006: 191-192). Es ist auch nicht zu leugnen, dass die Spendenbereitschaft der Großkapitalisten und ,Industriekapitäne ${ }^{6}$ um die Wende vom 19. zum 20. Jahrhundert den privaten Hochschulen einen Wettbewerbsvorteil bei der Neuausrichtung der Hochschullandschaft auf Forschung verschaffte, der diesen bei der Einwerbung von öffentlichen Forschungssubventionen 50 Jahre später zu Gute kommen sollte.

Ein großzügiges Steuerrecht und die ausgeprägte Tradition der Philanthropie können somit zu einer Erklärung der hohen Privatausgaben beitragen, sollten aber auch selbst Gegenstand eines umfassenden Erklärungsversuchs sein. Steuerpolitik ist wie andere Policies Ergebnis der Auseinandersetzung gesellschaftlicher Interessen mit unterschiedlichen Machtressourcen in einem bestimmten institutionellen Rahmen. Es muss somit um ein tieferes Verständnis der Ursachen des heutigen PolicyArrangements gehen. Der vorangehende Abschnitt hat deutlich gemacht, dass neben dem relativen Timing von makrosozialen Prozessen auch Regierungshandeln und -politik eine wichtige Rolle gespielt haben, die private und öffentliche Institutionen 
als gleichberechtigt anerkannten und damit die Position der privaten Hochschulen nachhaltig konsolidiert haben. Das klärt jedoch noch nicht die Frage, warum die Nachfrager nach Bildung (Studenten und ihre Eltern) dazu bereit waren und sind, hohe private Aufwendungen zu akzeptieren, wenn sie im Rahmen demokratischer Verfahren für stärker öffentliche und stärker subventionierte Bildungsalternativen votieren könnten. Die im letzten Abschnitt erläuterten Regierungspolitiken hätten langfristig keinen Bestand, wenn im Wahlvolk nicht eine grundlegende Übereinstimmung mit deren Effekten, d.h. der herausgehobenen Bedeutung von privaten Hochschulinstitutionen, vorhanden wäre. Daher wird nun als letztes Puzzleteil der Zusammenhang zwischen der Bildungspolitik und dem wohlfahrtsstaatlichen Umfeld in den Blick genommen.

\section{Das wohlfahrtsstaatliche Umfeld}

\subsection{Die Bedeutung für die Bildungspolitik}

Das wohlfahrtsstaatliche Umfeld prägt in entscheidender Weise die Verfügbarkeit von Optionen und Entscheidungsalternativen, die einem Individuum, das am Anfang seiner Bildungs- und Berufskarriere steht, zur Verfügung stehen. Im Hinblick auf die leitende Forschungsfrage sind zwei Elemente des wohlfahrtsstaatlichen Umfelds besonders hervorzuheben: Erstens trägt der liberale Arbeitsmarkt dazu bei, dass die ,Bildungsprämien' so hoch ausfallen, dass hohe Studiengebühren verkraftet werden können. Hohe Bildungsinvestitionen tragen langfristig auch $\mathrm{zu}$ wirtschaftlichem Wachstum und zur Entwicklung bei, so dass die ,anonyme Sozialpolitik des Marktmechanismus“ (Rosenberg 1967: 217) greifen kann. Zweitens drängt der schwach ausgeprägte soziale Schutz gegen Lebensrisiken wie Arbeitslosigkeit, Krankheit und zum Teil auch Alter Individuen mangels Alternative zu Investitionen in die eigene Bildung und Ausbildung.

Zunächst soll kurz die Bedeutung des liberalen Arbeitsmarktes erläutert werden. Erstens zeigt sich in den USA eine im OECD-Länder-Vergleich überdurchschnittlich deutliche Reduzierung der Arbeitslosenquoten beim Vergleich derjenigen ohne Sekundarschulabschluss mit denjenigen mit College-Abschluss. Die Quote reduziert sich von ca. $10 \%$ bei „High School Drop-Outs“ zu ca. $3 \%$ bei den College-Abgängern, also eine Reduzierung um ca. zwei Drittel (OECD 2005: 110). In den kontinentaleuropäischen Staaten fällt diese Reduktion nicht so deutlich aus (große Ausnahme: Deutschland und Österreich), wohl aber in Australien, Großbritannien und mit Einschränkungen in Kanada. Es finden sich somit erste Hinweise darauf, dass Investitionen in die eigene Bildung und Ausbildung in den Staaten mit liberalen Arbeitsmärkten einen effektiveren Schutz gegen Arbeitslosigkeit darstellen bzw. dass der Arbeitsmarkt eine Unterinvestition in das eigene Humankapital stärker bestraft.

Zweitens zeigt sich, dass der Zuwachs an Einkommen für Erwerbstätigkeit, der aus der zusätzlichen Investition in die eigene Bildung resultiert, besonders stark ausgeprägt ist. Auf einer Indexskala $(100=$ durchschnittliches Einkommen mit Sekundarschulabschluss) beträgt die Differenz in Indexpunkten zwischen einer Person ohne Sekundarschulabschluss und einer Person mit College-Abschluss („Tertiary Type A“) für die Vereinigten Staaten 125 Punkte (für 30-44-Jährige, vgl. OECD 
2005: 130). Lediglich in Großbritannien (111 Indexpunkte) ist die Bildungsprämie ähnlich hoch. ${ }^{8}$ Wenn die „Bildungsprämie“ (der Einkommenszuwachs aus der weiteren Investition in die eigene Bildung und Ausbildung) so hoch ist wie in den USA, wird dadurch ein effektiver Investitionsanreiz gesetzt. Hohe Studiengebühren stellen dann kein prohibitives Hindernis gegen Bildungspartizipation dar, wenn dem prospektiven Bildungsteilnehmer auf dem Kreditmarkt keine Hindernisse im Weg stehen, um den kurzfristigen Finanzierungsbedarf für Bildungsinvestitionen zu decken. Die öffentlichen Förderprogramme (siehe oben) sind ja auch auf die Behebung der kurzfristigen Kapitalmarkthindernisse ausgelegt.

Zur Erklärung der Forschungsfrage der hohen Privatausgaben muss aber eigentlich die Perspektive umgedreht werden: Hohe Studiengebühren werden deshalb toleriert (und bezahlt), weil die Aussicht auf eine Einkommenssteigerung durch zusätzliche Bildungsinvestitionen so hoch ist, dass sie die kurzfristig abschreckenden Effekte dominieren. Eine wichtige Rolle spielt hierbei auch die Stratifizierung des amerikanischen Hochschulsystems (Allmendinger 1989; Bastedo/Gumport 2003). In einem „General Skills“-System (Iversen 2005; Iversen/Soskice 2001) wie den USA kommt der Position einer Hochschule im nationalen Ranking eine wichtige Bedeutung zu. Solange private Hochschulen vordere Plätze im Ranking belegen, können sie sich auch höhere Studiengebühren leisten (vgl. auch Clotfelter 1996), weil sie den Absolventen gut bezahlte Jobs garantieren.

Als zweites Element des wohlfahrtsstaatlichen Umfeldes ist auf den schwach ausgeprägten Sozialschutz bei der Absicherung von Lebensrisiken wie Arbeitslosigkeit, Krankheit und - mit Einschränkungen - Alter hinzuweisen. Anstelle einer ausführlichen Darstellung dieses Sachverhaltes, der zu den besterforschten und bestbekannten Befunden der vergleichenden Wohlfahrtsstaatenforschung zählt, sei auf Kaufmann (2001: 827) verwiesen, der die Verleihung des Titels „Wohlfahrtsstaat“ an die USA als „durchaus fragwürdig“ bezeichnet. Die Konsequenz für die hier zu beantwortende Fragestellung ist, dass die Investition in die eigene Bildung und Ausbildung für viele nicht nur durch die hohen Bildungsprämien positiv motiviert ist, sondern vielfach auch die einzige Route zu besseren Jobs und damit zu einer besseren sozialen Absicherung darstellt. Wie zu Anfang bereits erwähnt, zeichnet sich der USamerikanische Wohlfahrtsstaat ja nicht nur durch den schwachen sozialen Schutz durch öffentliche sozialpolitische Institutionen aus, sondern durch die Schwerpunktsetzung auf private Alternativen (z.B. betriebliche Renten und Krankenversicherungen). Durch die Ausrichtung des Wohlfahrtsstaates auf private Sozialpolitik wird die Verbindung zwischen Erwerbstätigkeit und sozialem Schutz gestärkt - allerdings um den Preis einer höheren Einkommensungleichheit und einer höheren Ungleichheit in der Verteilung von Sozialleisungen (Hacker 2002: 24). Prospektive Bildungsteilnehmer werden somit durch die hohen Bildungsprämien des liberalen Arbeitsmarktes positiv zu Investitionen in Bildung motiviert, durch den schwachen sozialen Schutz durch öffentliche Sozialpolitik und die Ausrichtung des Wohlfahrtsstaates auf private Sozialpolitik aber auch dazu getrieben.

8 Zum Vergleich: Schweden: 61 Indexpunkte, Finnland: 77 Indexpunkte, Deutschland: 70 Indexpunkte, Kanada: 83 Indexpunkte, Australien: 57 Indexpunkte, aber Ungarn: 156 Indexpunkte (alle Daten aus OECD 2005: 130). 


\subsection{Sozialpolitische Folgewirkungen}

Wenn das wohlfahrtsstaatliche Umfeld Investitionen in die eigene Bildung und Ausbildung positiv (Bildungsprämien) und negativ (Absicherung) sanktioniert, so stellt sich zwangsläufig die Frage, inwiefern die Bildung in den USA sozialpolitische Funktionen, vor allem den Ausgleich von sozialer Ungleichheit, leisten kann, inwiefern die Bildung also bei der Umsetzung des amerikanischen Mythos vom unbeschränkten sozialen Aufstieg eine Rolle spielt.

Auf der Ebene der Motivationen und Perzeptionen der entscheidenden politischen Akteure jedenfalls taucht das Motiv des sozialen Ausgleichs und der Herstellung von Chancengleichheit immer wieder auf. Dies gilt vor allem für die Bildungsreformer des 19. und frühen 20. Jahrhunderts, die immer einen engen Zusammenhang zwischen Demokratisierung und Bildungsexpansion sahen und den paternalistischautokratischen Ansatz Preußens explizit ablehnten. Auch zu späteren Zeitpunkten ist immer wieder deutlich geworden, dass Bildung auch sozialpolitische Funktionen übernehmen sollte. Ein Beispiel hierfür ist der im Rahmen des „War on Poverty“ unter Präsident Johnson in den 1960er Jahren verabschiedete Elementary and Secondary Education Act, der nicht nur den Einfluss der Bundesebene auf die Primar- und Sekundarbildung ausdehnte, sondern Zuschüsse im Verhältnis zur Armutsquote auf Schuldistrikte verteilte (Ravitch 1983: 149). In den 1980er und 1990er Jahren wurde das Thema der Ungleichheit der Verteilung von Bildungsausgaben auf Schuldistrikte außerdem in einer Welle von Gerichtsentscheidungen auf der Ebene der Bundesstaaten behandelt, was oft umfangreiche Restrukturierungen der öffentlichen Schulfinanzierungssysteme zur Stärkung der Gleichverteilung zur Folge hatten (Wong 1999).

Auf der Ebene der gesellschaftlichen Realitäten muss der Befund allerdings gemischter ausfallen. Der liberale Arbeitsmarkt führt zwar dazu, dass für Absolventen von prestigeträchtigen Universitäten und Colleges die soziale Herkunft weniger prägekräftig ist als vielleicht in anderen Ländern. Die Problematik der ungleichen Zugangschancen zu Bildung greift allerdings zu einem früheren Zeitpunkt. Wegen der stark ausgeprägten Dezentralisierung des Primar- und Sekundarschulwesens schlägt sich die soziale Herkunft stärker über indirekte Faktoren wie die Schul- und Wohnortwahl nieder. Öffentliche Schulen werden immer noch zu einem großen Teil über lokal erhobene Grundsteuern (,property tax “) finanziert, so dass es eine starke Korrelation zwischen dem Wohlstand in einem Schuldistrikt (Immobilienwerte) und der Ausstattung seiner Schulen gibt. Die Wahl einer Schule innerhalb des Distriktes ist normalerweise nicht frei, sondern wird von der lokalen Schulverwaltung festgelegt und ist damit zu einem wesentlichen Teil vom Einkommen der Eltern abhängig. Der Erwerb bestimmter akademischer und nicht-akademischer Qualitäten und Qualifikationen kann aber bereits auf Primar- und frühem Sekundarniveau entscheidend sein für den späteren Zugang zu Elite-Hochschulinstitutionen. Dies ist besonders dann relevant, wenn, wie Guillory (2000: 1157) argumentiert, die Sekundarschulen tendenziell auf Kosten der Universitäten vernachlässigt werden und nur diejenigen an hoch selektive Eliteinstitutionen gelangen, die sich durch private Initiative oder elterlichen Wohlstand den Besuch einer hoch-qualitativen Sekundarschule ermöglicht haben (vgl. Windolf 1990: 104).

In den letzten Jahrzehnten sind, auch wegen der erwähnten Welle von Gerichtsentscheidungen, einige Veränderungen zu beobachten, die aber nicht eindeutig in die 
Richtung einer Verbesserung der sozialen Ausgleichsfunktion laufen. Einige Bundesstaaten haben mehr Wahlmöglichkeiten geschaffen und den Griff der lokalen Schuladministrationen gelockert. Außerdem gibt es Reformbewegungen und -bestrebungen, die zur Errichtung von Charter Schools geführt haben. Hierbei handelt es sich um Schulen, die zwar von öffentlichen Geldern finanziert werden, aber von einem Großteil der öffentlichen Regulationen ausgenommen und in der Trägerschaft unabhängig von den lokalen Schuladministrationen sind (Bierlein 1997). Unter dem Strich dürften die Bestrebungen zur Schaffung von mehr Wahlmöglichkeiten nicht die weniger Einkommensstarken begünstigt haben, die dadurch in die Lage versetzt worden wären, ihre Kinder zu besseren Schulen in andere Distrikte zu schicken. Vielmehr sind wahrscheinlich diejenigen besser gestellt worden, die nach einer möglichst kostengünstigen Möglichkeit gesucht haben, ihre Kinder aus den wenig angesehenen öffentlichen Schulen herauszubekommen. Eine Untersuchung des bildungspolitischen Meinungsdiskurses hat nachgewiesen, dass schon in den 1980er Jahren ein Wandel im Diskurs von Fragen der Ressourcengleichverteilung hin zu Fragen der Bildungsqualität zu beobachten ist (Marshall et al. 1989: 13). Dieser Befund stützt die eben geäußerte Vermutung.

Das generell offenere und liberale Bildungs- und Arbeitsmarktregime der Vereinigten Staaten eröffnet dennoch Räume für individuelle Initiative, die im vergleichsweise stark an Klassenlagen orientierten deutschen System weniger vorhanden sind (Allmendinger 1999: 48). Dies kann am Beispiel der Bildungssituation der Immigranten verdeutlicht werden. Choy (2002: 31) zeigt, dass der Immigrantenstatus, wenn anfängliche Hürden, die vor allem durch die missliche wirtschaftliche Lage der meisten Immigranten erzeugt werden, überwunden sind, keinen merklichen Einfluss hat auf den weiteren Bildungsweg der Immigrantenkinder. White und Glick (2000) zeigen, dass für Immigrantenkinder die Wahrscheinlichkeit, die High School erfolgreich abzuschließen, sogar höher ist als für andere Bevölkerungsgruppen, allerdings nur, wenn man im Rahmen von multivariaten statistischen Analysen den Einfluss von sozio-ökonomischen Faktoren berücksichtigt. In anderen Worten formuliert: Die scheinbare ungünstige Lage der Immigranten im Bildungssystem ist vor allem eine Konsequenz der ungünstigen sozio-ökonomischen Umstände, unter denen diese Bevölkerungsgruppe lebt, und nicht eine Folge ihres Immigrantenstatus per se. Bemerkenswert ist außerdem, dass die Wahrscheinlichkeit, nach der High School eine postsekundäre Bildung zu erlangen, für Immigranten aus der zweiten Generation höher ist als für Angehörige der dritten Generation, die schon stärker „amerikanisiert“ sind (White/Glick 2000: 685).

Ein vorläufiges Fazit im Hinblick auf die Frage der Übernahme von sozialpolitischen Funktionen, vor allem den Ausgleich von sozialer Ungleichheit, durch die Bildung fällt folgendermaßen aus: Die persistenten Ungleichheiten in der Verteilung von Bildungschancen in der Primar- und Sekundarbildung, die mit Ungleichheiten in Einkommen, sozialer Herkunft und Erwerbsstatus korrelieren, sind Folge des dezentralen Charakters des US-amerikanischen Bildungssystems. Offene Diskriminierungen im Zugang zu Hochschulbildung sind selten anzutreffen, aber die erwähnten indirekten Diskriminierungen führen dazu, dass Bildung nicht systematisch als universaler Mechanismus zur Herstellung von Chancengleichheit agieren kann. Im Rahmen des breitmaschigen sozial- und bildungspolitischen Netzes verbleiben allerdings 
Spielräume für individuelle Initiative, die von Hochmotivierten genutzt werden können, wenn sie es schaffen, die anfänglichen Hürden eines verminderten sozialen Status zu überwinden.

\section{Zusammenfassung und Fazit}

Die zentrale Fragestellung des vorliegenden Aufsatzes war die Erklärung der herausgehobenen Stellung privater Bildungsinstitutionen in den USA, die sich vor dem Hintergrund des internationalen Vergleichs, aber auch des Vergleichs mit der Entwicklung im Primar- und Sekundarschulwesen gezeigt hat. Zunächst wurde argumentiert, dass in der frühen Entwicklungsphase des US-amerikanischen Bildungs- und Sozialstaates Anfang und Mitte des 19. Jahrhunderts von den entscheidenden politischen Akteuren der Bildung emanzipative, demokratieförderliche und sozialpolitische Funktionen zugedacht wurden. Die schwach ausgeprägte Zentralisierung der Verwaltungsbürokratie hat zu einer Vermischung der öffentlichen und privaten Verantwortung für Bildungsinstitutionen sowie zu einer engen Anbindung an die lokale oder weltanschauliche Gemeinde geführt. Im Laufe der Zeit wurde der Trennstrich zwischen öffentlichen und privaten Schulen aber immer stärker gezogen, was langfristig allerdings zur Folge hatte, dass private Schulen zunehmend von öffentlichen verdrängt wurden. Im Hochschulbereich waren ähnliche Verdrängungstendenzen erkennbar wie im Primar- und Sekundarschulbereich, d.h. langfristig hätte es auch hier zu einer Dominanz der öffentlichen über die privaten Institutionen kommen müssen. Der Faktor, der den Vormarsch der öffentlichen Institutionen im Hochschulbereich aufgehalten oder zumindest gebremst hat, ist Regierungshandeln und -politik (vor allem der Bundesregierung). Durch die Bindung der Studienförderung an Individuen und nicht an Institutionen sowie durch eine Forschungsförderungspolitik, die zwischen privaten und öffentlichen Institutionen nicht unterscheidet, hat sich die Position der privaten Hochschul-Bildungsinstitutionen auf einem höheren Niveau konsolidiert als im Primar- und Sekundarschulwesen. Des Weiteren wurde auf die Bedeutung des liberalen Arbeitsmarktes verwiesen, der hohe Bildungsprämien erzeugt und damit hohe Studiengebühren und hohe Investitionen in die eigene Bildung und Ausbildung rechtfertigt. Hinzu kommt ein schwacher sozialer Schutz durch öffentliche Sozialpolitik und die an den Status der Erwerbstätigkeit anknüpfende private Sozialpolitik, die Unterinvestitionen in Bildung negativ sanktionieren.

\section{Literaturverzeichnis}

Adema, Willem (2001): Net Social Expenditure, 2nd Edition. OECD Labour Market and Social Policy - Occasional Papers No. 52, Paris.

Allmendinger, Jutta (1989): „Educational Systems and Labour Market Outcomes“, European Sociological Review 5/3: 231-250.

Allmendinger, Jutta (1999): „Bildungsarmut: Zur Verschränkung von Bildungs- und Sozialpolitik", Soziale Welt 50: 35-50. 
Allmendinger, Jutta; Leibfried, Stephan (2002): „Bildungsarmut im Sozialstaat“, in: Günter Burkart; Jürgen Wolf (Hg.): Erkundungen zur Soziologie der Generationen. Opladen: Leske + Budrich, 287-315.

Bastedo, Michael N.; Gumport, Patricia J. (2003): „Access to what? Mission differentiation and academic stratification in U.S. public higher education", Higher Education 46: 341-359.

Beckert, Jens (2004): Unverdientes Vermögen: Soziologie des Erbrechts. Frankfurt a.M., New York: Campus.

Berube, Maurice R. (1994): American School Reform: Progressive, Equity and Excellence Movements, 1883-1993. Westport, CT u.a.: Praeger.

Bierlein, Louann A. (1997): „The Charter School Movement“, in: Diane Ravitch; Joseph P. Viteritti (Hg.): New Schools for a New Century: The Redesign of Urban Education. New Haven u.a.: Yale University Press, 37-60.

Bragg, Debra (2001): „Community College Access, Mission, and Outcomes: Considering Intriguing Intersections and Challenges“, Peabody Journal of Education 76/1: 93-116.

Brubacher, John S.; Willis, Rudy (1997): Higher Education in Transition: A History of American Colleges and Universities. New Brunswick u.a.: Transaction Publishers.

Busemeyer, Marius R. (2006): Die Bildungsausgaben der USA im internationalen Vergleich: Politische Geschicbte, Debatten und Erklärungsansätz̧e. Wiesbaden: Deutscher Universitäts-Verlag.

Chambers, M. M. (1962): „Legal Developments in Higher Education, 1862-1962“, in: William W. Brickman; Stanley Lehrer (Hg.): A Century of Higher Education: Classical Citadel to Collegiate Colossus. Westport, CT: Greenwood Press, 32-49.

Choy, Susan P. (2002): Access \& Persistence: Finding from 10 Years of Longitudinal Research on Students. Washington, D.C.: American Council on Education.

Choy, Susan P. (2004): Paying for College: Changes between 1990 and 2000 for Full-Time Dependent Undergraduates, Findings from The Condition of Education 2004. National Center for Education Statistics, U.S. Department for Education, Institute of Educational Sciences, Washington, D.C.

Church, Robert L. (1976): Education in the United States: An Interpretive History. London u.a.: Free Press, Macmillan.

Clotfelter, Charles T. (1996): Buying the Best: Cost Escalation in Elite Higher Education. Princeton: Princeton University Press.

Coleman, James S.; Hoffer, Thomas (1987): Public and Private High Schools: The Impact of Communities. New York: Basic Books.

Cremin, Lawrence A. (1976): Traditions of American Education. New York: Basic Books.

Dichanz, Horst (1991): Schulen in den USA: Einheit und Vielfalt in einem flexiblen Schulsystem. Weinheim u.a.: Juventa Verlag.

Dresselhaus, Günter (1997): Das deutsche Bildungswesen zwischen Tradition und Fortschritt - Analyse eines Sonderwegs. Münster: LIT.

Esping-Andersen, Gøsta (1990): The Three Worlds of Welfare Capitalism. Princeton, NJ: Princeton University Press.

Fenton, Richard J.; Gardner, John; Singh, Sandeep (2001): „Rethinking Cuts in Public Higher Education: An American Example“, Education Economics 9/1: 53-68.

Guillory, John (2000): „The System of Graduate Education“, PMLA 115/5: 1154-1163.

Hacker, Jacob S. (2002): The Divided Welfare State: The Battle over Public and Private Social Benefits in the United States. Cambridge: Cambridge University Press. 
Heidenheimer, Arnold J. (1981): „Education and Social Security Entitlements in Europe and America“, in: Peter Flora; Arnold J. Heidenheimer (Hg.): The Development of Welfare States in Europe and America. New Brunswick u.a.: Transaction Books, 269-304.

Iversen, Torben (2005): Capitalism, Democracy, and Welfare. Cambridge: Cambridge University Press.

Iversen, Torben; Soskice, David (2001): „An Asset Theory of Social Policy Preferences“, American Political Science Review 95/4: 875-893.

Jencks, Christopher; Riesman, David (2002[1968]): The Academic Revolution. New Brunswick u.a.: Transaction Publishers.

Jorgenson, Lloyd P. (1987): The State and the Non-Public School, 1825-1925. Columbia: University of Missouri Press.

Kaestle, Carl F. (1983): Pillars of the Republic: Common Schools and American Society. New York: Hill and Wang.

Kaufmann, Franz-Xaver (2001): „Der deutsche Sozialstaat im internationalen Vergleich“, in: Franz-Xaver Kaufmann; Bundesarchiv; Bundesministerium für Arbeit und Sozialordnung (Hg.): Geschichte der Sozialpolitike in Deutschland seit 1945, Band 1. Frankfurt a.M.: Suhrkamp, 799-987.

Leibfried, Stephan; Castles, Francis G.; Obinger, Herbert (2005): „,Old` and ,new politics` in federal welfare states", in: Herbert Obinger; Stephan Leibfried; Francis G. Castles (Hg.): Federalism and the Welfare State: New World and European Experiences. Cambridge et al.: Cambridge University Press, 307-355.

Lucas, Christopher J. (1994): American Higher Education: A History. New York: St. Martin's Press.

Marshall, Catherine; Mitchell, Douglas; Wirt, Frederick (1989): Culture and Education Policy in the American States. New York u.a.: Falmer Press.

McKeown-Moak, Mary P. (2001): Financing Higher Education in the New Century: The Third Annual Report from the States. State Higher Education Executive Officers, Denver, CO.

Münch, Joachim (2000): „Die Hochschulsysteme Deutschlands, der USA und Japans im Vergleich"; in: Robert von Weizsäcker (Hg.): Schul- und Hocbschulorganisation. Berlin, 75-116.

Nasaw, David (1979): Schooled to Order: A Social History of Public Schooling in the United States. New York: Oxford University Press.

NCES, National Center for Education Statistics (2005): Digest of Education Statistics. National Center for Education Statistics. Download unter: http://nces.ed.gov/ programs/digest/ (Zugriff am 12. Januar 2007).

NSF, National Science Foundation (2004): Academic Research and Development Expenditures: Fiscal Year 2004. National Science Foundation. Download unter: http:// www.nsf.gov/statistics/nsf06323/tables.htm (Zugriff am 12. Januar 2007).

Obinger, Herbert; Castles, Francis G.; Leibfried, Stephan (2005): „Introduction: Federalism and the welfare state"; in: Herbert Obinger; Stephan Leibfried; Francis G. Castles (Hg.): Federalism and the Welfare State: New World and European Experiences. Cambridge et al.: Cambridge University Press, 1-48.

Obinger, Herbert; Wagschal, Uwe (Hg.) (2000): Der gezügelte Woblfahrtsstaat: Sozialpolitik in reichen Industrienationen. Frankfurt a.M. u.a.: Campus.

OECD, Organisation for Economic Co-operation and Development (2005): Education at a Glance: OECD Indicators. Paris: OECD.

OECD, Organisation for Economic Co-operation and Development (2006a): Education at a Glance: OECD Indicators. Paris: OECD. 
OECD, Organisation for Economic Co-operation and Development (2006b): OECD Factbook 2006. Paris: OECD.

Peters, B. Guy (2004): American Public Policy: Promises and Performance. Washington, D.C.: CQ Press.

Pfnister, Allan O. (1962): „A Century of Church-Related College“, in: William W. Brickman; Stanley Lehrer (Hg.): A Century of Higher Education: Classical Citadel to Collegiate Colossus. Westport, CT: Greenwood Press, 80-93.

Ralph, John H.; Rubinson, Richard, (1980): „Immigration and the Expansion of Schooling in the United States, 1890-1970“, American Sociological Review 45/6: 943-954.

Ravitch, Diane (1983): The Troubled Crusade: American Education, 1945-1980. New York: Basic Books.

Rosenberg, Hans (1967): Grosse Depression und Bismarckzeit: Wirtschaftsablauf, Gesellschaft und Politik in Mitteleuropa. Berlin: Walter de Gruyter.

Schmidt, Manfred G. (2005): Sozialpolitik in Deutscbland: Historische Entwicklung und internationaler Vergleich. Wiesbaden: VS Verlag für Sozialwissenschaften.

Schneider, Reinhart (1982): „Die Bildungsentwicklung in den westeuropäischen Staaten 18701975“, Zeitschrift für Soziologie 11/3: 207-226.

Skocpol, Theda (1992): Protecting Soldiers and Mothers: The Political Origins of Social Policy in the United States. Cambridge, MA u.a.: Belknap Press of Harvard University Press.

Titmuss, Richard (1958): Essays on the Welfare State. London: Allen and Unwin.

Veysey, Laurence R. (1965): The Emergence of the American University. Chicago u.a.: The University of Chicago Press.

White, Michael J.; Glick, Jennifer E. (2000): „Generation Status, Social Capital, and the Routes out of High School“, Sociological Forum 15/4: 671-691.

Windolf, Paul (1990): Die Expansion der Universitäten 1870-1995: Ein internationaler Vergleich. Stuttgart: Enke.

Wong, Kenneth K. (1999): Funding Public Schools: Politics and Policies. Lawrence, KA: University Press of Kansas.

Wright, Karen (2002): „Generosity vs. Altruism: Philanthropy and Charity in the US and UK“, Voluntas 12/4: 399-416.

Korrespondenz:

E-Mail:
Dr. Marius R. Busemeyer

Max-Planck-Institut für Gesellschaftsforschung

Paulstr. 3

50676 Köln

busemeyer@mpifg.de 